\title{
Towards a stable and absolute atmospheric carbon dioxide instrument using spectroscopic null method
}

\author{
B. Xiang ${ }^{1}$, D. D. Nelson ${ }^{2}$, J. B. McManus ${ }^{2}$, M. S. Zahniser ${ }^{2}$, and S. C. Wofsy ${ }^{1}$ \\ ${ }^{1}$ School of Engineering and Applied Science and Department of Earth and Planetary Sciences, Harvard University, \\ Cambridge, MA 02138, USA \\ ${ }^{2}$ Aerodyne Research, Inc., 45 Manning Road, Billerica, MA 01821-3976, USA \\ Correspondence to: B. Xiang (bxiang@seas.harvard.edu)
}

Received: 9 February 2013 - Published in Atmos. Meas. Tech. Discuss.: 22 February 2013

Revised: 22 May 2013 - Accepted: 4 June 2013 - Published: 5 July 2013

\begin{abstract}
We present a novel spectral method to measure atmospheric carbon dioxide $\left(\mathrm{CO}_{2}\right)$ with high precision and stability without resorting to calibration tanks during longterm operation. This spectral null method improves precision by reducing spectral proportional noise associated with laser emission instabilities. We employ sealed quartz cells with known $\mathrm{CO}_{2}$ column densities to serve as the permanent internal references in the null method, which improve the instrument's stability and accuracy. A prototype instrument - ABsolute Carbon dioxide (ABC) is developed using this new approach. The instrument has a one-second precision of $0.02 \mathrm{ppm}$, which averages down to $0.007 \mathrm{ppm}$ within one minute. Long-term stability of within $0.1 \mathrm{ppm}$ is achieved without any calibrations for over a one-month period. These results have the potential for eliminating the need for calibration cylinders for high accuracy field measurements of carbon dioxide.
\end{abstract}

\section{Introduction}

Knowledge on the global budget and the transport of atmospheric carbon dioxide $\left(\mathrm{CO}_{2}\right)$ is essential to understanding the climate change effects introduced by this important greenhouse gas (GHG). Improvements in the measurement capabilities of $\mathrm{CO}_{2}$ instruments can help to achieve this scientific goal. Among various instrument requirements, accuracy and precision are always the top priority and serve as the basis of meaningful scientific measurements and interpretations. Since most of the global $\mathrm{CO}_{2}$ observation network monitoring stations are at remote measurement sites, the in- strument's capabilities of in-field measurements, long-term unattended operation and stability are important concerns. In addition, regional flux studies involving tower or aircraft observations as well as eddy covariance flux measurements require real-time, fast response and automated operations.

In recent years, various $\mathrm{CO}_{2}$ instruments have been developed in laboratories or made commercially available that are useful for advanced atmospheric science studies. Instrument techniques that have been used include tunable infrared laser differential absorption spectroscopy (TILDAS) (Tittel et al., 2003; Wofsy et al., 2011), non-dispersive infrared absorption (NDIR) spectroscopy (Komhyr et al., 1989; Stephens et al., 2011), integrated cavity output spectroscopy (ICOS) (Baer et al., 2002), cavity-enhanced absorption spectroscopy (CEAS) (Engeln et al., 1998; Rella et al., 2013), FT-IR spectroscopy (Esler et al., 2000; Griffith et al., 2012), photoacoustic detection (Andersen et al., 2010), etc. Based on these technologies, $\mathrm{CO}_{2}$ instruments with high precision have been improved in terms of compactness, automation, low cost and survival under extreme environmental conditions. However, one common disadvantage with most previous instruments is that calibration tanks are employed and the majority of them cannot claim good long-term (over months) stability without relying on these standards.

Calibration tanks are commonly employed in all stable atmospheric trace gas measurements in order to correct for instrument drifts and to assure measurement accuracies. The "tank science" required to achieve high accuracy is labor intensive and has no generally accepted protocol, which adds another level of uncertainty to the measurements. Taking the case of $\mathrm{CO}_{2}$ monitoring as an example, calibration tanks 
throughout the world are filled with compressed air and calibrated with World Meteorology Organization Global Atmosphere Watch program (WMO GAW) standards at the National Oceanic and Atmospheric Administration Earth System Research Laboratory Global Monitoring Division (NOAA ESRL GMD) Central Calibration Lab (CCL) in order to enable accurate and intercomparable $\mathrm{CO}_{2}$ dry air mole fraction measurements (Zhao and Tans, 2006). Individual laboratories then use the calibrated tanks from NOAA CCL to calibrate their own secondary and even tertiary sets of tanks for field or lab measurements. Various compressed air sources (e.g., natural or combustion) can have dramatic differences in isotopic composition which further complicates the calibration procedure (this is further discussed in Sect. 3.3). The reported $\mathrm{CO}_{2}$ mixing ratios from calibration tanks are significantly dependent on the individual tank conditions (e.g., fill gas source, impurities, usage, leakage, position, tank material, regulator characteristics), the environmental conditions (e.g., temperature, humidity, pressure), as well as personal operation experiences and skills (Keeling et al., 2007). A blind intercomparison test within WMO standard calibration tanks showed up to $0.2 \mathrm{ppm}$ (parts per million dry air mole fraction) $\mathrm{CO}_{2}$ mixing ratio difference within the 340 and $380 \mathrm{ppm}$ range (Zhou et al., 2009). For ecosystem carbon flux estimation, this measurement bias at one site location can introduce an error estimation of $\sim 50 \mathrm{~g} \mathrm{C} \mathrm{m}^{-2} \mathrm{yr}^{-1}$ at another site $500 \mathrm{~km}$ away, a number that is nearly half of the annual ecosystem flux maximum at this spatial scale (Stephens et al., 2011). To get rid of this labor intensive and unstable standard tank problem, we test the idea of employing a permanent and stable internal spectroscopic reference source, as an included feature of the spectroscopic null method, to replace the calibration tanks. However, stable gas standards will be employed, at a much reduced frequency and with great care, as a practical approach for quality assurance.

We have developed a laboratory prototype $\mathbf{A B}$ solute $\mathbf{C O}_{2}$ spectrometer $(\mathrm{ABC})$ capable of measuring ambient air $\mathrm{CO}_{2}$ mixing ratio with precision of $0.02 \mathrm{ppm}$ at $1 \mathrm{~Hz}$, and with long-term stability of $0.1 \mathrm{ppm}$ on the timescale of a month, without resorting to any calibration tanks during long-term unattended operation. These performances put the $\mathrm{ABC}$ instrument among the best quality $\mathrm{CO}_{2}$ instruments currently available, and yet with the unique feature of not requiring calibration tanks. The technically novel features of this instrument are the spectroscopic null method and the use of a permanently sealed quartz cell as the internal standard. The null method improves the measurement precision by enabling the instrument to be much less sensitive to any spectral proportional noise, such as absorption line strength change (e.g., with temperature), laser tuning rate drift, laser multi-modes, optics temperature variation and spectral fitting routine uncertainty (Nelson et al., 2008). The sealed quartz cell improves the measurement accuracy and longterm stability by providing a known column density of $\mathrm{CO}_{2}$ as an internal reference which is invariant over time or under environment condition changes. This work successfully demonstrates the spectroscopic null scheme and the use of a permanent internal reference for instrument stability improvements. The method also has the potential to be adopted on various spectrometers measuring stable atmospheric species without external calibrations. Following this successful laboratory demonstration, we are currently developing a field-deployable $\mathrm{ABC}$ instrument with many design improvements.

\section{Experimental details}

The fundamental technique we use for measuring ambient $\mathrm{CO}_{2}$ mixing ratio is Tunable Infrared Laser Differential Absorption Spectrometry (TILDAS). Details of the instrument design as well as the data acquisition and analysis have been described before (McManus et al., 2006; Nelson et al., 2006). In general, we scan a tunable IR laser over the desired wavelength region and pass the laser light through a multi-pass sample cell at reduced pressure. The sample absorption is measured via nonlinear least square Voigt fitting (Olivero and Longbothum, 1977) using simulated transition lines with known spectral parameters (line shape and position from HITRAN, and pressure and temperature from measurements) to obtain a quantitative mixing ratio. The experimental setup of $\mathrm{ABC}$ is distinct in the way that we aim to measure the nearly nulled ratio spectra between sample and reference paths. In this way the signal to noise ratio (SNR) and long-term stability are greatly improved. The high performance of $\mathrm{ABC}$ also relies on a stable distributed feedback (DFB) diode laser, two sensitive and cryogen-free InAs detectors, an effective thermal control scheme for the optics and stable temperature and pressure measurements. In this section, we discuss in detail the special features of this instrument as compared to the other TILDAS spectrometers previously developed in our laboratory.

\subsection{Null method}

The null method has been employed by many spectroscopic instruments, such as NDIR analyzers and other quantum cascade laser spectrometers (QCLS) that we have developed. It helps reduce instrument noise associated with the finite depth of the absorption line, which we describe as "proportional noise" in the spectral analysis (i.e, noise that is proportional to the mixing ratio). Many kinds of instrument instabilities can affect the experimental spectrum and challenge the spectral fitting routine. Noise sources can be separated into two groups: baseline noise and proportional noise. Common sources of baseline noise are optical fringes, external beam absorption, spectral interferences from other species, laser power variation and electronic noise. Potential sources of proportional noise or drift include laser tuning rate, line 
width and mode purity variations, detector linearity variation, numerical noise in the spectral analysis algorithm, and errors in the measurement of the sample pressure, temperature and optical path length. Most spectroscopy instruments rely on frequent background and calibration gases to separately minimize these two types of noise.

For many of the spectroscopic instruments that we have developed, laser emission stability has been found to be a major instrument drift source. The laser instabilities come from electrical noise in the current controller or the modulation circuit, and from the temperature control of the laser. Both the baseline and absorption line shape are affected. In previous designs we have used laser power normalization and differential absorption methods to compensate for these drift sources. For single cell measurements, a secondary beam from the same laser may be employed to normalize the sample beam (i.e., the sample transmission spectrum is divided by that of the reference; this is also called the ratio or residual spectrum) to minimize the laser power variation effect on the baseline (Nelson et al., 2008). The null method further advances this solution by matching the sample and reference absorptions from a single laser, which largely cancels the effects of laser output variations on the measurements.

The null method is a variant of the TILDAS approach. Based on the Beer-Lambert law and the ideal gas law, the sample $\mathrm{CO}_{2}$ mixing ratio $\left(\mathrm{MR}_{\mathrm{s}}\right)$ from differential measurements can be approximately expressed as

$$
\begin{aligned}
\mathrm{MR}_{\mathrm{s}} & =\operatorname{MR}_{\mathrm{r}}\left(T_{\mathrm{s}} / T_{\mathrm{r}}\right)\left(\sigma_{\mathrm{r}, T} / \sigma_{\mathrm{s}, T}\right)\left(P_{\mathrm{r}} / P_{\mathrm{s}}\right)\left(L_{\mathrm{r}} / L_{\mathrm{s}}\right) \\
& +A_{\mathrm{n}}\left(k_{\mathrm{B}} T_{\mathrm{s}} / \sigma_{\mathrm{s}, T} P_{\mathrm{s}} L_{\mathrm{s}}\right),
\end{aligned}
$$

where $\mathrm{MR}_{\mathrm{r}}$ is the reference gas mixing ratio, $T, P$ and $L$ are temperature, pressure and optical path length, $\sigma$ is the temperature dependent integrated absorption line cross section, $A_{\mathrm{n}}$ is the integrated absorbance of the normalized spectrum, $k_{\mathrm{B}}$ is the Boltzman's constant, subscripts of "s" and " $\mathrm{r}$ " denote sample and reference parameters separately. Ideally, if temperature and pressure between the sample and the reference cell are carefully maintained to be the same, absorption line strength and line width are the same for both spectra. The first term of Eq. (1) is simplified to $\mathrm{MR}_{\mathrm{r}} * L_{\mathrm{r}} / L_{\mathrm{s}}$, which is considered as the equivalent reference gas mixing ratio $\left(\mathrm{MR}_{\mathrm{r} \text {, eq }}\right)$, as if the reference gas is measured in the sample cell. Equation (1) can then be transformed as

$\mathrm{MR}_{\mathrm{s}}=\mathrm{MR}_{\mathrm{r}, \text { eq }}+A_{\mathrm{n}}\left(k_{\mathrm{B}} T_{\mathrm{s}} / \sigma_{\mathrm{s}, T} P_{\mathrm{s}} L_{\mathrm{s}}\right)$.

We fill reference cells with a column density of $\mathrm{CO}_{2}$ close to the equivalent for ambient $\mathrm{CO}_{2}$ in the sample cell, so the absorption from the normalized spectrum is only a small fraction of that from the reference cell spectrum. The closer the sample and the reference absorption are to equal, the less proportional noise interference on the normalized spectrum is observed. An ideal case is a complete null (flat) spectrum where $A_{\mathrm{n}}=0$ and the second term in Eq. (1) is zero. As a result, knowledge of laser parameters such as tuning rate, mode purity and line width for spectral fitting are no longer needed. The sample mixing ratio is exactly the equivalent reference mixing ratio.

The realization of the null method has raised some operational challenges. The first step is to balance the temperature and the pressure in the sample and the reference cell. Optical path lengths of the sample and the reference cell need to be accurately known in order to convert the absolute reference mixing ratio to the equivalent one in the sample cell. Although on a much smaller absorption level, the residual spectrum must still be measured accurately and the spectral fitting analysis should be able to simulate the relatively small absorption signal even in the presence of small pressure or temperature differences between the cells. Unequal ambient $\mathrm{CO}_{2}$ absorption along the external paths of the sample and the reference line will cause baseline curvature in the normalized spectrum which is likely to change with time, if not purged or balanced by the path lengths. Thermally driven optical fringes of a strength and free spectral range that can influence the measurement may be present in the optical system, so that careful thermal control of the optics is prudent. We targeted all the above issues in the development of $\mathrm{ABC}$ and demonstrate that the null method can be realized with good instrument performance.

\subsection{Sealed quartz reference cell}

The accuracy and the long-term stability performance of $\mathrm{ABC}$ are assured by permanently sealed quartz reference cells containing known column densities of $\mathrm{CO}_{2}$ in dry air. Column density is the product of the $\mathrm{CO}_{2}$ number density $\left(N_{\mathrm{r}}\right)$ and the optical path length $\left(L_{\mathrm{r}}\right)$ of the quartz cell. We chose quartz as the cell material due to its chemical inactiveness, low surface interactions, low thermal expansion coefficient $\left(\sim 10^{-6} / \mathrm{K}\right)$, good IR transmission (to $\sim 3 \mu \mathrm{m}$ ) and the capability to be sealed permanently. Once filled and sealed, the number density $\left(N_{\mathrm{r}}\right)$ inside the quartz cell is a fixed number, invariant to changes in environmental conditions such as pressure and temperature. This is also true for the column density $\left(N_{\mathrm{r}} L_{\mathrm{r}}\right)$ provided that sufficiently stable laser beam alignment is maintained through the cell.

The sealed quartz cells are cylindrical cells with quartz to pyrex graded seal tubes. Figure 1 shows the picture of an unsealed custom made quartz cell (Spectrocell Inc, USA). The optical path length along the cell axis is $10 \mathrm{~cm}$ and the window outer diameter is $2.2 \mathrm{~cm}$. Cells were encased in plastic and metal tubes for both protection and passive thermal control. The enclosures were clamped onto metal block supporters bolted down on the optical table for position fixation and good thermal contact. Irises were installed on both sides of the metal enclosure to facilitate the beam alignment through the center of the cell and to ensure a stable optical path length $\left(L_{\mathrm{r}}\right)$. We made several trials placing the quartz cell into the system and re-aligning with the help of irises. No optical path length change was observed based on the reported stable $\mathrm{CO}_{2}$ 


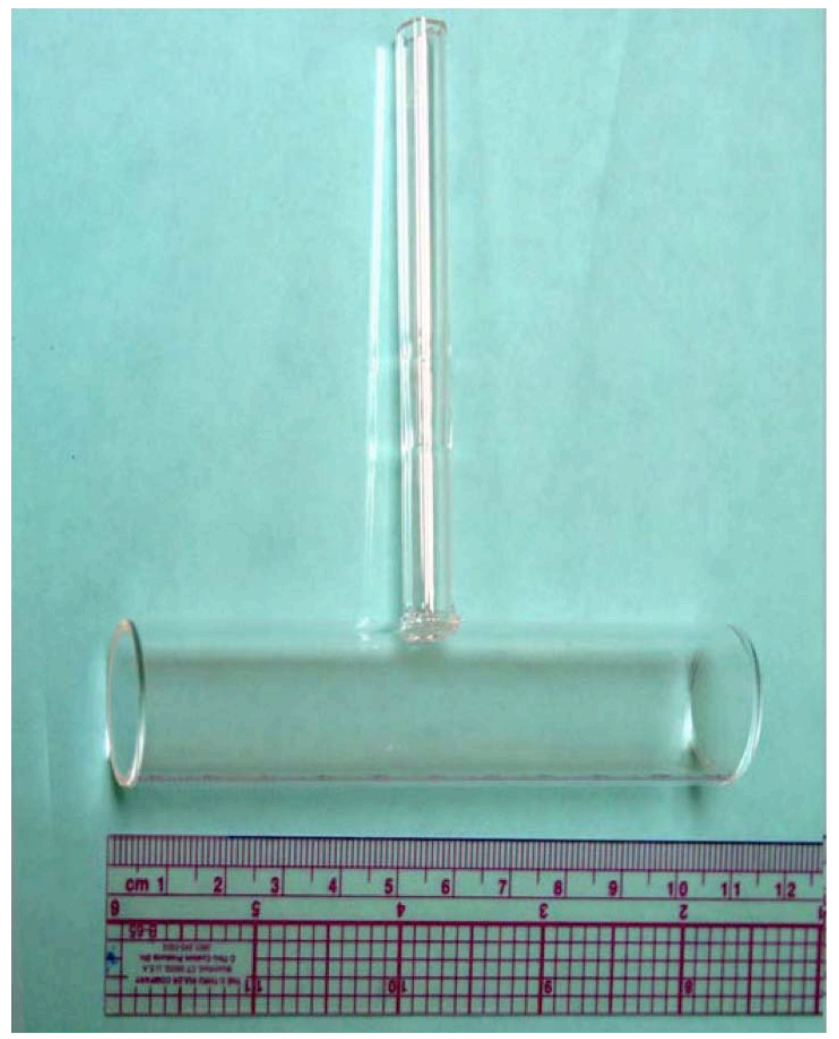

Fig. 1. Unsealed quartz reference cell. The seal tube has graded transitions from quartz to pyrex, where the cell is sealed. The optical path length along the cell axis is $10 \mathrm{~cm}$ and the window outer diameter is $2.2 \mathrm{~cm}$. The cell front window was tilted by $1^{\circ}$ angle with respect to the incoming beam.

mixing ratio. The cell front window was tilted by $1^{\circ}$ angle with respect to the incoming beam to avoid back-reflection and optical fringes generated on the window surface. This adds a small uncertainty on $L_{\mathrm{r}}$ when the laser beam is slightly mis-aligned. In order to achieve an accuracy level of $0.1 \mathrm{ppm}$ $\mathrm{CO}_{2}$ (1 in 4000 of the ambient mixing ratio), the tolerable mis-alignment distance from the center of the front and the back windows should be no more than $1.12 \mathrm{~mm}$, which can be achieved optically and mechanically.

Two quartz cells were filled with $4 \% \mathrm{CO}_{2}$ (in volume, from a pure $\mathrm{CO}_{2}$ tank source) in a total of $0.7 \mathrm{kPa}$ (5 Torr) dry, $\mathrm{CO}_{2}$-free air. We select this low pressure to balance between pressure broadening and absorption depth. The sample cell is operated at the same pressure. Its optical path length $\left(L_{\mathrm{S}}\right)$ is $10.16 \mathrm{~m}$, about 100 times that of the quartz cell. As a result, the equivalent $\mathrm{CO}_{2}$ mixing ratio in the quartz cell is around $400 \mathrm{ppm}$, close to the ambient level. The cells were pumped with high vacuum for several days before fillings. After they were filled, we monitored the cell pressure change and the spectrum at an adjacent water absorption region for days to make sure there was no internal water desorption. Then the cells were sealed by a glass-blower on the pyrex

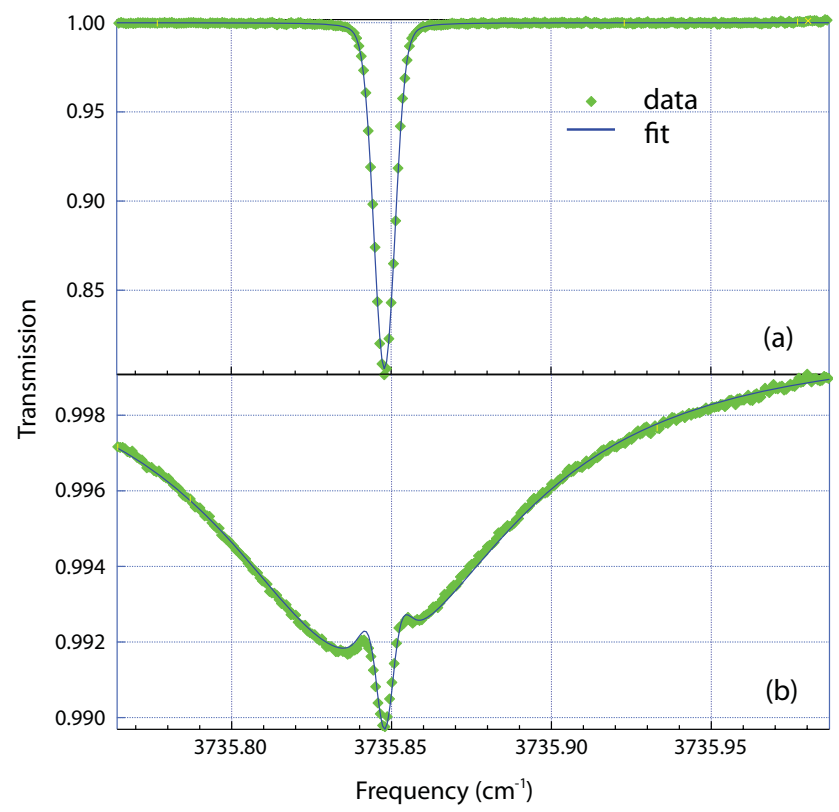

Fig. 2. Transmission spectra of (a) single reference beam and (b) normalized sample beam (sample spectrum divided by reference spectrum). The reference gas mixing ratio was similar to but smaller than that of the sample gas, resulting in a negative residual spectrum (sharp peak) with 100 times smaller absorbance than that from the single beam spectrum. The broad spectral feature shown in (b) appears to be due to $\mathrm{CO}_{2}$ absorption within the sample detector sealed enclosure.

side tube in a quick motion and were left leak free. The final $\mathrm{CO}_{2}$ mixing ratio and the cell pressure can vary slightly from the predetermined value due to the sealing process. We estimated the sealed pressure by comparing the experimental line width with the simulation, which should be accurate within $13 \mathrm{~Pa}(0.1$ Torr). This is a sensitive measurement, and the cells used in this study have an internal pressure of 5.5 Torr at $296 \mathrm{~K}$. The extra pressure compared to the fill value can be explained mostly by the pressure gradient generated around the seal point from the hot torch flame, and partly by the small zero offset on the pressure sensor. Quartz cell pressures were re-visited $2 \mathrm{yr}$ after the seal and no significant changes were observed at the same wavelength and temperature, indicating essentially no leaks within this long time period. The sealed $\mathrm{CO}_{2}$ column density or the effective mixing ratio in the quartz cells need to be measured only one time using known calibration standards.

\subsection{Spectrometer setup}

Figure 2a shows a single beam transmission spectrum from a sealed quartz reference cell. We choose the nearIR ${ }^{12} \mathrm{C}^{16} \mathrm{O}_{2}$ absorption line at $3735.8480 \mathrm{~cm}^{-1}$, the $\mathrm{R}(30) \mathrm{e}$ $10011 \leftarrow 00001$ transition, as the instrument working wavelength. This selection is based on considerations of the laser and detector qualities, absorption line strength and its 

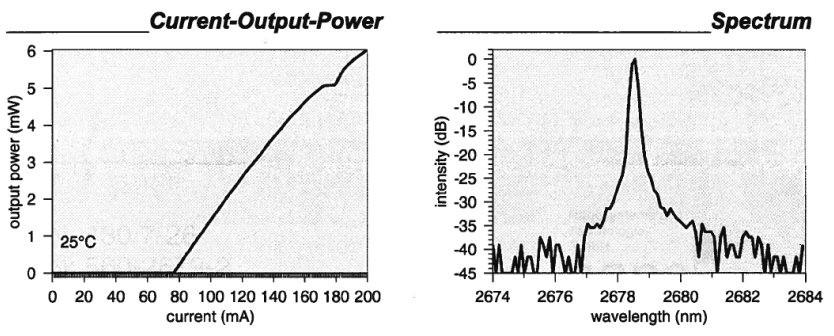

Fig. 3. DFB diode laser characteristics and spectrum.

temperature dependence, quartz window transmittance, sample cell mirror reflectivity and spectral interferences from other species and from the external path $\mathrm{CO}_{2}$ absorption. A $2.7 \mu \mathrm{m}$ Nanoplus DFB diode laser is operated at room temperature (typically $298 \mathrm{~K}$ ) and is tuned by a programmed current ramp to wavelength scan a narrow region of $0.3 \mathrm{~cm}^{-1}$ across the chosen transition. Its maximum operation voltage is $2 \mathrm{~V}$ and the maximum operation current is $200 \mathrm{~mA}$. Figure 3 shows the laser current-output power curve and its spectral purity. The typical tuning rate behavior of this laser is $-0.28 \mathrm{~cm}^{-1} \mathrm{~K}^{-1}$ by temperature and $-0.03 \mathrm{~cm}^{-1} \mathrm{~mA}^{-1}$ by current. The laser line width is $<0.001 \mathrm{~cm}^{-1}$, much smaller than the Doppler broadening of the absorption line. Output power of the laser is $\sim 2 \mathrm{~mW}$ and no multi-mode behavior is observed at the working wavelength. The mode purity was determined by adding sufficient $\mathrm{CO}_{2}$ to the sample cell to absorb $100 \%$ of the light and the residual transmission was $<10^{-3}$ at the line center. Sample and reference beams are detected separately by two two-stage thermoelectric (TE) controlled InAs photodiode detectors (Teledyne Judson Technologies, USA) with good specific detectivity $\left(6.5 \times 10^{10} \mathrm{~cm} \mathrm{~Hz}^{1 / 2} \mathrm{~W}^{-1}\right)$ and linearity. Detector saturation effects were not significant, as the reported $\mathrm{CO}_{2}$ mixing ratio did not depend upon the laser output power. The absorption line strength of the chosen transition from the HITRAN08 database is $3.024 \times 10^{-20} \mathrm{~cm}^{-1} /$ (molecule $\mathrm{cm}^{-2}$ ), which gives rise to $20 \%$ absorbance on the single beam spectrum (see Fig. 2a). The selected transition has relatively smaller lower state energy compared to adjacent lines according to HITRAN and results in a small temperature dependence of the line strength, $0.08 \% \mathrm{~K}^{-1}$ at $296 \mathrm{~K}$. The quartz cell window transmission of $90 \%$ and the multi-pass cell mirror reflectivity of $99.2 \%$ assure small fractional light losses. The selected wavelength region is free of water absorption.

The optical module is based on a $60 \times 30 \mathrm{~cm}^{2}$ breadboard and a diagram of the setup is shown in Fig. $4 a$. Figure $4 b$ shows a photograph of the optical table.

The diode laser is positioned in a commercial Thorlabs laser housing (purple) and its output first gets collected and re-focused by a $15 \times$ reflecting objective mounted on a threeaxis translation stage allowing for fine focus adjustments. A pin-hole between the objective and the first reflecting mirror
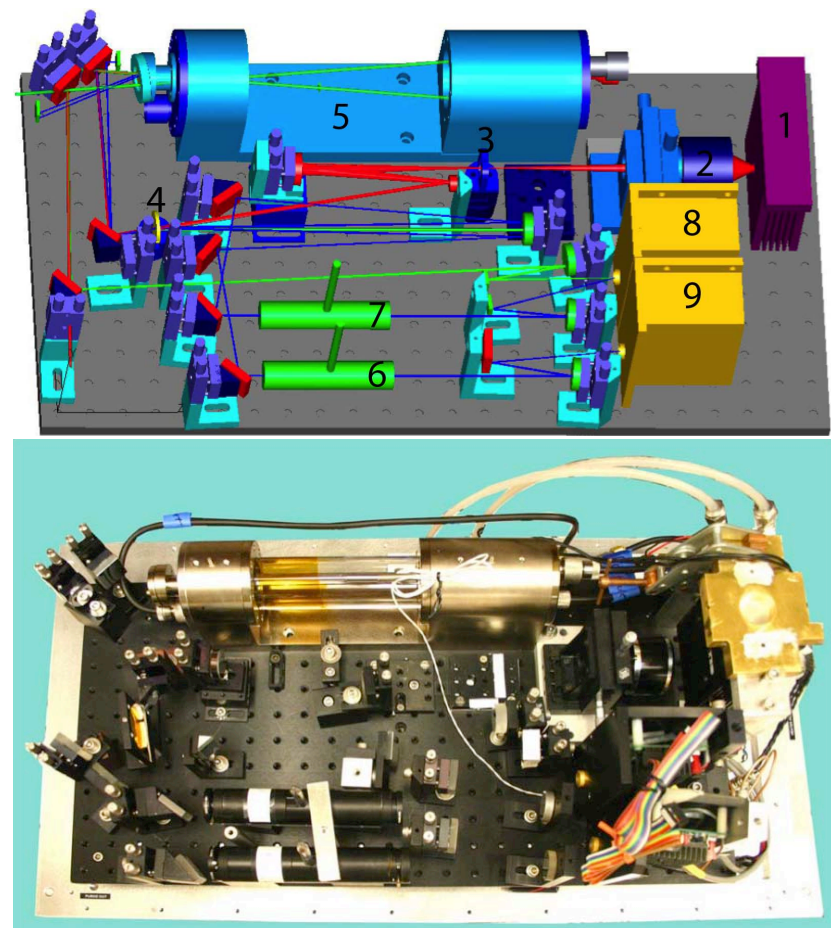

Fig. 4. ABC-prototype instrument optical layout. (a) design: 1 DFB diode laser; $2-15 \times$ reflective objective; 3 - pin hole; 4 beam splitter; 5 - sample cell; 6 - primary quartz reference cell; 7 secondary quartz reference cell; 8 - sample detector; 9 - reference detector. (b) photograph of the optical table.

serves as a reference point for alignment, with the help from a visible trace laser. The main beam (red) is separated by a wedged beam splitter (yellow) into three parts: the transmitted beam goes into the sample multi-pass cell and gets detected by the sample detector (yellow); the front and the back reflected beams are directed into separate quartz reference cells (green) and detected by the sample and the reference detectors separately. The sample cell is an Aerodyne astigmatic Herriott cell (AMAC-36) with an internal volume of $\sim 0.3 \mathrm{~L}$. The absorption path length between the cell mirrors is set to $10.05 \pm 0.01 \mathrm{~m}$. This is achieved by adjusting the cell mirror separation $(20.09 \mathrm{~cm})$ and the twist angle $\left(15.1^{\circ}\right)$ to obtain a 50-pass "incomplete" reflection pattern (McManus, 2007; McManus et al., 2011). The extra absorption path between the cell front window and front mirror is included in the total path.

The quartz cell at the outer path in the setup picture serves as the major reference cell. The sample beam and the inner path quartz cell beam share the same detector. A shutter is installed to select between these two. The secondary quartz cell enables the instrument to check and characterize any system drifts in the sample detector and in the major quartz reference beam. External path lengths for these three beams are closely matched to minimize the effect of external-path $\mathrm{CO}_{2}$. Dry and $\mathrm{CO}_{2}$-free air is employed to purge the optical box at 
a slow flow rate to further reduce this potential interference. Purge gas is inconvenient in terms of remote operations and temperature disturbances. We plan to improve the hermetic sealing of the optical box, chemically scrub and homogenize external-path $\mathrm{CO}_{2}$, and further balance the external beam paths in the next development step.

\subsection{Spectral analysis}

The spectral analysis of the normalized or nulled spectrum is complicated by the fact that there will inevitably be small pressure and temperature differences between the sample cell and the sealed reference cell. These will cause line shape differences between the sample and reference spectra and hence will induce a second derivative shape in the normalized spectrum near the null point. This effect will, of course, increase the residuals in the spectral fit if not included in the analysis. Hence, our spectral analysis software has been extended to do so. Rather than analyzing the null spectrum as a single Voigt line shape, it is analyzed as a ratio of Voigt lines whose shapes are determined by the sample pressure and temperature for one line and the reference pressure and temperature on the other.

An example of a null spectrum is shown in Fig. 2b. The reference gas mixing ratio was similar to that of the sample. Thus the ratio spectrum has much smaller absorbance than either the sample or reference spectrum. The sharp, low pressure absorption feature in the plot is the normalized sample spectrum with an absorbance of $\sim 2 \times 10^{-3}$ which is 100 times smaller than that from a single beam spectrum (Fig. 2a). At the time of measurement in Fig. 2b, there is more absorbance in the sample cell than in the quartz cell, resulting in a negative residual spectrum. The sample gas pressure was smaller than that of the quartz cell. Hence, the sharp feature shows a clear second derivative shape which is well described by the blue fit line from the spectral model.

The sharp spectral line is superimposed on a much broader spectral feature. This is clearly the same $\mathrm{CO}_{2}$ line but observed near atmospheric pressure and shifted to lower frequency due to pressure induced shift. This feature was unexpected and appears to be due to $\mathrm{CO}_{2}$ absorption within the sample detector sealed enclosure. It is not due to $\mathrm{CO}_{2}$ in the external optical path since it was not reduced by purging the optical compartment with dry nitrogen. In addition, we tried switching the sample and the reference detectors and found that the broad absorption feature is associated with the sample detector. The reference detector does not show this feature so the feature does not get canceled out in the null spectrum. This extra $\mathrm{CO}_{2}$ in the sample detector is estimated to have much higher mixing ratio than the ambient level and was relatively stable over the course of this study. We account for this broad absorption by fitting it separately using the Voigt model with fixed mixing ratio and pressure (one atmosphere). The latest version of the $\mathrm{ABC}$ instrument platform has two new detectors installed and is free of such interference.

\subsection{Control and measurement of pressure and temperature}

Long-term absolute $\mathrm{CO}_{2}$ measurements require accurate pressure and temperature measurements. In order to achieve an accuracy of $0.1 \mathrm{ppm} \mathrm{CO}_{2}$, the sample pressure reading should be stable within 1 part in 4000 of 5 Torr which is 1.25 mTorr. We use a 10 Torr capacitance manometer (MKS 122A) to monitor the sample cell pressure. The sensor is placed inside the temperature controlled optical box to reduce thermal drift and is connected to the sample cell through a short tube to reduce dead volume. In this work, all flowing measurements were made within 0.1 Torr of the 5 Torr set point and the calibration measurements were performed in cycles which required only a few hours, relaxing the requirements on the pressure sensor. Hence, the results reported here are not subject to errors caused by inaccuracy or drift in the pressure sensor. However, long-term measurements using this approach would require a pressure sensor with longterm drift less than 1.25 mTorr at 5 Torr. Currently, we are conducting long-term accuracy tests (zero- and span-drift) on three more pressure sensors: MKS 626B, MKS 627D and MKS 722A, all in the 10 Torr range.

Temperature influences the instrument's stability through a variety of mechanisms including phase motion of optical fringes. Fringes are frequently a problem in laser spectrometers. Their free spectral range (FSR) can be large, depending on the source resonator separations in the optical system. There may exist several fringes of different FSR and the combined effects further complicate the baseline structure on the spectrum (Werle, 2011). Fringes with FSR comparable to the sample absorption line width can bias the spectral fitting results and their movements with time can affect the instrument's short-term and long-term stability. We made several efforts to suppress fringe effects. They include careful optical design and alignment, fixing every component tightly, using mirrors with high reflectivity, using the astigmatic multi-pass cell, tilting quartz cell windows, wiggling some optical components, temperature and current stabilizing the laser, locking the laser emission line stringently, as well as averaging high frequency fringe features on the baseline. We also tried fitting the interfering fringe with a sine wave and following its movement. The fringe effects were greatly suppressed after all the above efforts.

We improved the temperature stability of the optics by adopting a re-circulating liquid coolant scheme instead of the electronic heating scheme originally used. The old control scheme involves active heating of the optical box several degrees above the environment temperature, with a slightly higher set point for the box cover than for the bottom plate in order to generate a vertical temperature gradient to suppress convection. The heat dissipation from the laser and detectors 


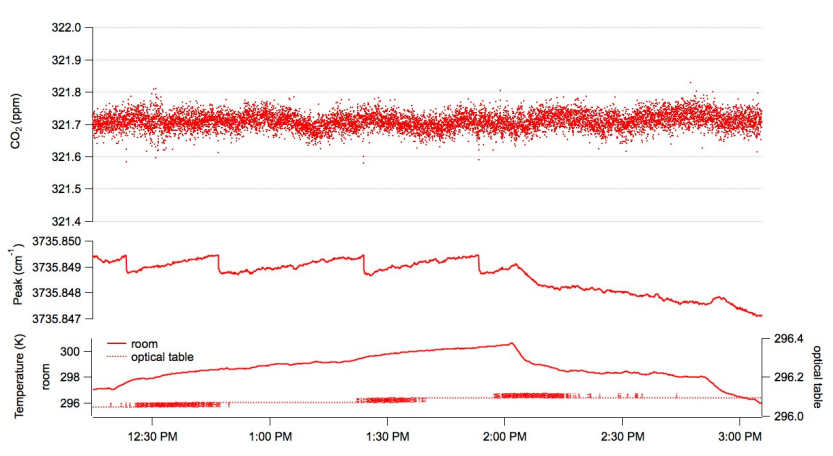

Fig. 5. Short-term stability test of ABC. A secondary sealed quartz cell was measured using the null method at $1 \mathrm{~Hz}$ for $3 \mathrm{~h}$, under rapid room temperature change of $5 \mathrm{~K}$ magnitude. Bottom panel: room temperature (scale on the left) and optics temperature (a much smaller scale on the right); middle panel: $\mathrm{CO}_{2}$ absorption line peak position, indicating the laser frequency drift as a result of the temperature variation; top panel: measured equivalent $\mathrm{CO}_{2}$ mixing ratio from the secondary sealed quartz cell.

inside the enclosure complicates the thermal distribution. We have tested and demonstrated that the liquid cooling scheme results in better thermal control of the optical box. The liquid output from a chiller, ThermoCube 300 (Solid State Cooling Inc, USA), first goes to the laser peltier, then to several cooling plates attached beneath the bottom of the optical table. Due to the large thermal capacity of the coolant and the tight connection of all optics to the table, the dissipated heat from the internal heat sources is removed quickly and effectively. The chiller output temperature is set at room temperature $(296 \mathrm{~K})$ to minimize thermal gradients. We thermally isolated the optical box with $5 \mathrm{~cm}$ polystyrene foam (thermal resistance of $2 \mathrm{~K} \mathrm{~m}^{2} \mathrm{~W}^{-1}$ ). This control scheme reduces optical table temperature variation to 1 part in 100 of the room temperature variation. The sample gas gets temperature stabilized by first going through a long copper coil inside the optical box. The sample and the quartz cells were designed to be strongly thermally coupled to the optical table and were therefore assumed to be at the same temperature. Any temperature differences were certainly small and their impact was minimized by the small temperature dependence of the absorption line strength $\left(0.08 \% \mathrm{~K}^{-1}\right.$ at $\left.296 \mathrm{~K}\right)$.

\section{Instrument performance}

\subsection{Null effect}

The null method and the improved temperature stability greatly improve the instrument precision and short-term stability. One example of this improvement is a $3 \mathrm{~h}$ temperature experiment. In this test we chose to measure the spectral ratio of the two sealed quartz cells. Since both the sample and the reference quartz cells were filled at the same time through an Ultra-Torr Tee connector (Swagelok), the $\mathrm{CO}_{2}$ mixing ra-

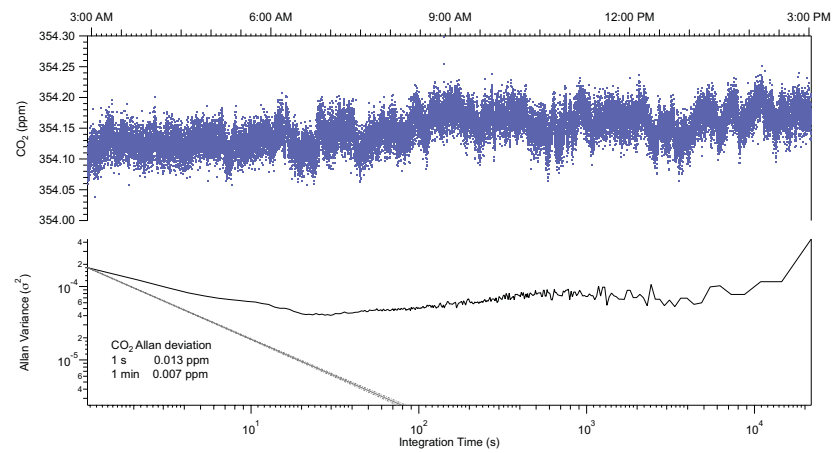

Fig. 6. Instrument performance of measuring a tank source for $12 \mathrm{~h}$ continuously using the null method. Top panel shows the real-time $1 \mathrm{~Hz}$ data; bottom panel is the Allan variance plot showing the instrument's one-second precision of $0.013 \mathrm{ppm}$, and $0.007 \mathrm{ppm}$ with one minute averaging.

tios in these two cells are almost the same, giving rise to a nearly nulled ratio spectrum. During the $3 \mathrm{~h}$ measurement period, we challenged the instrument with rapid room temperature variations up to $5 \mathrm{~K}$. The room temperature profile is shown in the bottom panel of Fig. 5 with its scale on the left. Optics temperature was measured at the same time and its profile is shown also in the bottom panel of Fig. 5 with a much smaller scale on the right. Room temperature variation caused the laser frequency to drift subtly, demonstrated by the absorption peak position change (in $\mathrm{cm}^{-1}$ ) shown in the middle panel of Fig. 5. The measured equivalent $\mathrm{CO}_{2}$ mixing ratio from the sample quartz cell is shown at the top panel of Fig. 5. All measurements were at $1 \mathrm{~Hz}$.

The temperature control scheme worked well to maintain the optical table temperature within $\pm 20 \mathrm{mK}$. We did not observe drifts in the reported $\mathrm{CO}_{2}$ mixing ratio. There were four extreme conditions where TDLWintel (control software) corrected the laser frequency by changing the laser Peltier set temperature. Even during these sharp frequency corrections, the changes in the corresponding $\mathrm{CO}_{2}$ mixing ratio are well below the noise level. The one-second precision during this measurement is $0.023 \mathrm{ppm}$.

Null measurements for flowing gas samples were also performed and were observed to have even better precisions, an example of which is shown as an Allan variance (Werle et al., 1993) plot in Fig. 6. In this case, the gas sample from a medical grade compressed natural air tank (Middlesex Gas Inc, USA) was passed through the sample system at a flow rate of $400 \mathrm{sccm}$ using a critical orifice and was measured continuously for $12 \mathrm{~h}$.

The short-term noise of $0.013 \mathrm{ppm}(0.04 \%$ ) at one second measurement interval is excellent. It does not average down as random noise but is rather limited by the instrument drift (Werle, 2010). We do not fully understand the source of the drift, probably its source is electronic or optical. However, such drift was bounded and the instrument accuracy was 


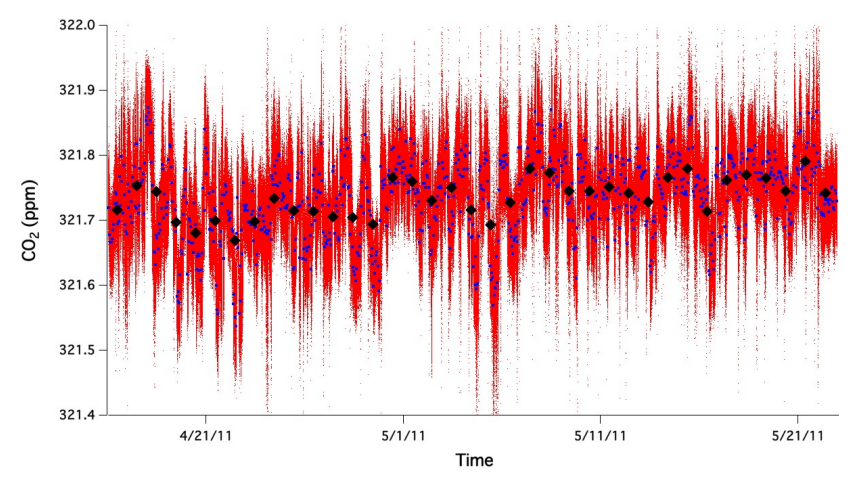

Fig. 7. One month continuous monitor of the $\mathrm{CO}_{2}$ mixing ratio from a second sealed quartz cell without any calibration or operational intervention. The overall temperature change during the measurement month was $\sim 20 \mathrm{~K}$. Sample rate is $1 \mathrm{~Hz}$. Real-time data are shown in red without any averaging. The blue dots and the black diamonds are calculated hourly and daily averaged values. They show essentially no $\mathrm{CO}_{2}$ drift within one-month period and less than $0.1 \mathrm{ppm}$ day-to-day $\mathrm{CO}_{2}$ variations. The one-sigma standard deviation is $0.059 \mathrm{ppm}$ for the hourly mean and is $0.031 \mathrm{ppm}$ for the daily mean.

maintained within $0.1 \mathrm{ppm}$ throughout the measurement period, as shown in Fig. 6. The associated absorbance precision is $8 \times 10^{-6}$ (with $20 \%$ absorbance on a single cell spectrum, see Fig. 2a), which corresponds to an absorbance per unit length of $8 \times 10^{-9} \mathrm{~cm}^{-1}$ for the $10.16 \mathrm{~m}$ sample cell.

\subsection{Long-term (one month) stability}

The long-term stability of the $\mathrm{ABC}$ instrument was demonstrated by running it continuously and unattended for over one month. We selected the second quartz cell as the sample in this measurement in order to prevent any possible sample drifts introduced by tank handling and consumption. An alternate way to demonstrate the instrument's long-term stability with flowing gas samples would be periodic checking of the same high pressure cylinder with extremely careful gas handling, including adequate flushing of the sample line, withdrawing air at high pressure level and control of the cylinder temperature. The diurnal room temperature variation in the laboratory is typically around $10 \mathrm{~K}$ and the overall temperature change during the measurement month was $\sim 20 \mathrm{~K}$.

Figure 7 shows long-term measurement result of $\mathrm{CO}_{2}$ mixing ratio at $1 \mathrm{~Hz}$ between 16 April and 22 May 2011 without any calibration or operational intervention. We calculated the hourly and daily averaged values through the whole dataset to reveal the long-term trend. The results show essentially no drift within one-month period and less than $0.1 \mathrm{ppm}$ day-to-day $\mathrm{CO}_{2}$ variations, indicating that the null method effectively reduces long-term measurement drift. The onesigma standard deviation is $0.059 \mathrm{ppm}$ for the hourly averages, and is $0.031 \mathrm{ppm}$ for the daily averages. This quartz cell comparison demonstrates the instrument's potential capability to achieve measurement accuracy which meets the WMO Data Quality Objectives ( $\pm 0.1 \mathrm{ppm}$ in Northern Hemisphere) without the use of field calibration.

\subsection{Potential accuracy}

Direct calibration of the $\mathrm{ABC}$ instrument with primary standards is the ideal approach to instrument calibration. It should be more accurate than calibration with secondary standards and would have the added benefit that the primary standards are typically supplied with a well-known isotopic composition. Hence, it would be the preferred approach for one-time calibration of the commercial instrument at the time of production. However, the calibration approach tested in this work was comparison to secondary standards which are tied to primaries by a NDIR measurement. We adopted this approach because we wanted to be able to monitor and verify the instrument frequently and for an extended time period. Moreover, this indirect approach is the method that most end users would be likely to choose to verify instrument performance. In this section we show an example of how to compare the measurements between a Licor instrument (broad band absorption) and $\mathrm{ABC}$ (single peak absorption).

We evaluated the instrument accuracy using three certified tanks with $\mathrm{CO}_{2}$ mixing ratio ranging from 338 to $403 \mathrm{ppm}$, a span of $65 \mathrm{ppm}$ around the typical ambient level. These three tanks were prepared separately by Scott-Marrin and Scott Specialty gas companies, and were calibrated by a NDIR analyzer (Licor 6251) using primary standards at Harvard University traceable to the WMO Central Calibration Lab (CCL) $\mathrm{CO}_{2}$ standards.

We used one of these tanks to calibrate the quartz reference cell, and measured the other two using the $\mathrm{ABC}$ instrument. We sampled these tanks alternately to test the instrument's response at different $\mathrm{CO}_{2}$ levels. Measurements were made for at least $5 \mathrm{~min}$ and were repeated 5-10 times for each tank. The calibration and measurement results for the tanks are listed in Tables 1 and 2 and the detailed explanation follows.

$\mathrm{CO}_{2}$ mixing ratios from the $\mathrm{ABC}$ measurements and from the Licor calibrations cannot necessarily be directly compared at the $0.1 \mathrm{ppm}$ accuracy level because of potential variation in the isotopic compositions of the calibration gases. $\mathrm{ABC}$ probes a single absorption line of the major isotopologue ${ }^{12} \mathrm{C}^{16} \mathrm{O}_{2}$ (hereafter referred to as 626 ), and hence directly measures the 626 mole fractions. $\mathrm{ABC}$ can report the total $\mathrm{CO}_{2}$ mixing ratio by assuming that the sample gas has a known isotopic composition. This is completely acceptable for atmospheric composition measurements where the isotopic composition of background air does not vary enough to be significant. However, calibration gases can have significantly different isotopic compositions which could prevent the $\mathrm{ABC}$ instrument from reporting the total $\mathrm{CO}_{2}$ mixing ratio with $0.1 \mathrm{ppm}$ accuracy when measuring a calibration tank with unknown isotopic composition. 
Table 1. Calibration tank $\mathrm{CO}_{2}$ information. Errors are 1 standard deviation from repeated measurements.

\begin{tabular}{lcccrr}
\hline $\begin{array}{l}\text { Tank } \\
\text { ID }\end{array}$ & Raw $\mathrm{CO}_{2} *(\mathrm{ppm})$ & $\delta^{13} \mathrm{C}^{* *}(\% \circ)$ & $\delta^{18} \mathrm{O}^{* *}(\%)$ & $\begin{array}{r}\text { Corrected total } \\
\mathrm{CO}_{2} * * *(\mathrm{ppm})\end{array}$ & $\begin{array}{r}626 * * * * \\
(\mathrm{ppm})\end{array}$ \\
\hline$\# 1$ & $338.072 \pm 0.006$ & $-39.9 \pm 0.46$ & $1.98 \pm 0.2$ & 338.0150 & 332.8332 \\
$\# 2$ & $346.728 \pm 0.011$ & $-49.4 \pm 0.3$ & $35.2 \pm 0.2$ & 346.6295 & 341.3062 \\
$\# 3$ & $402.937 \pm 0.006$ & $-36.7 \pm 0.35$ & $4.26 \pm 0.17$ & 402.8773 & 396.6835 \\
\hline
\end{tabular}

* The raw values of $\mathrm{CO}_{2}$ mole fractions in tanks were measured by modified Licor 6251 (Daube et al., 2002) and calibrated against tanks (filled with natural air) traceable to the NOAA total $\mathrm{CO}_{2}$ standards. Measurements were repeated twice for each tank. ** Isotopic compositions of $\mathrm{CO}_{2}$ in the tanks were calibrated by the Aerodyne $\mathrm{QCLS}_{-} \mathrm{CO}_{2}$ isotope instrument, referencing to a standard tank characterized by IRMS at the Stable Isotope Ratio Facility for Environmental Research at the University of Utah. $\delta^{13} \mathrm{C}$ values are on the VPDB scale (R13_VPDB $=0.0111797$ ), $\delta^{18} \mathrm{O}$ values are on the VSMOW scale $(\mathrm{R} 18$ _VSMOW $=0.0020052)$. *** The total $\mathrm{CO}_{2}$ were calculated by correcting the tank $\mathrm{CO}_{2}$ isotopic compositions for those in natural air $\left(-10 \%\right.$ for $\delta^{13} \mathrm{C}$ and $40 \%$ for $\left.\delta^{18} \mathrm{O}\right)$, and by correcting the Licor responses using mean RMR values (1.00565, 0.33 and 1.345 for 626,636 and 628) from the two Li-6252 instruments studied in Tohjima et al. (2009). **** The fraction of total $\mathrm{CO}_{2}$ that is 626 (F626) was calculated as $\mathrm{F} 626=\mathrm{F} 16 * \mathrm{~F} 12 * \mathrm{~F} 16 \approx[1 /(1+\mathrm{R} 18+\mathrm{R} 17)] *[1 /(1+\mathrm{R} 13)] *[1 /(1+\mathrm{R} 18+\mathrm{R} 17)] . \mathrm{F} 16$ is the fraction of total oxygen that is ${ }^{16} \mathrm{O} ; \mathrm{F} 12$ is the fraction of total carbon that is ${ }^{12} \mathrm{C} . \mathrm{R} 18=\left(\delta^{18} \mathrm{O}+1\right) * \mathrm{R} 18 \_\mathrm{VSMOW} ; \mathrm{R} 13=\left(\delta^{13} \mathrm{C}+1\right) *$ R13_VPDB. We assume the R17 value from VSMOW (0.0003799) for all three tanks since we did not measure them.

Table 2. ABC-prototype accuracy performance. All reported values are for the major $\mathrm{CO}_{2}$ isotopologue 626 , in units of ppm. Errors are 1 standard deviation from repeated measurements.

\begin{tabular}{|c|c|c|c|c|c|c|c|}
\hline \multicolumn{2}{|c|}{ Cal tank (Licor) } & \multicolumn{3}{|c|}{ Sample tank (ABC) } & \multicolumn{3}{|c|}{ Bias (ABC-Licor) } \\
\hline & & $\# 1$ & $\# 2$ & \#3 & $\# 1$ & $\# 2$ & $\# 3$ \\
\hline$\# 1$ & $332.833 \pm 0.006$ & NA & $341.214 \pm 0.06$ & $396.906 \pm 0.02$ & NA & $-0.09 \pm 0.06$ & $0.22 \pm 0.02$ \\
\hline$\# 2$ & $341.306 \pm 0.011$ & $332.926 \pm 0.06$ & NA & $396.844 \pm 0.09$ & $0.09 \pm 0.06$ & NA & $0.16 \pm 0.09$ \\
\hline$\# 3$ & $396.684 \pm 0.006$ & $332.611 \pm 0.02$ & $341.146 \pm 0.09$ & NA & $-0.22 \pm 0.02$ & $-0.16 \pm 0.09$ & NA \\
\hline
\end{tabular}

The response of the Licor instrument is more complex. The Licor uses an optical band-pass filter designed to transmit primarily the absorption band of 626 . However, absorption bands from the other $\mathrm{CO}_{2}$ isotopologues shift to lower wavenumbers and are partially included in the transmission region. The effect of the isotopic composition on the Licor response is dependent on the filter position, which can vary from instrument to instrument even within the same Licor model. Tohjima et al. (2009) quantified the theoretical relative molar response (RMR) of three Licor analyzers (Li6252, Li-6252, and Li-6262) to each isotopologue based on the infrared absorbance by the individual isotopologues. In order to compare the measurements made by Licor and $\mathrm{ABC}$, we use the RMRs provided by Tohjima et al. (2009) to correct the Licor measurements for isotopic variation and to express the results as measurements of the primary (626) isotopologue of $\mathrm{CO}_{2}$. This requires knowledge of the isotopic composition of the calibration gases and the RMRs.

The isotopic compositions of $\mathrm{CO}_{2}\left(\delta^{13} \mathrm{C}\right.$ and $\left.\delta^{18} \mathrm{O}\right)$ in the three tanks were measured using an Aerodyne quantum cascade laser- $\mathrm{CO}_{2}$ isotope (QCL_CO $\mathrm{CO}_{2}$ iso) instrument and were calibrated against a standard tank characterized by IRMS at the Stable Isotope Ratio Facility for Environmental Research at the University of Utah. Isotopic values in the three tanks are listed in Table 1. We used these values to convert the Licor reported values to 626 mole fractions in two steps. First we converted the "raw" total $\mathrm{CO}_{2}$ mixing ratios in column 1 to corrected total $\mathrm{CO}_{2}$ mixing ratios in column 5. Column 6 reports the 626 mixing ratio which follows directly from column 5 and the known isotopic abundances.

To obtain the corrected total $\mathrm{CO}_{2}$ mixing ratios in Table 1 (column 5), we used the mean of the RMR values for the two Li-6252 instruments studied in Tohjima et al. (2009) and made corrections for ${ }^{12} \mathrm{C}^{16} \mathrm{O}_{2}(626),{ }^{13} \mathrm{C}^{16} \mathrm{O}_{2}$ (636) and ${ }^{12} \mathrm{C}^{16} \mathrm{O}^{18} \mathrm{O}$ (628) only. We calculate that an unrecognized isotopic composition difference of $76 \%$ in $\delta 13 \mathrm{C}$ from the natural air $(\delta 13 \mathrm{C}=-10 \%$ ) would result in a $0.1 \mathrm{ppm}$ error in the reported value of the [626]. Similarly, an unrecognized isotopic shift of $46 \%$ in $\delta 180$ from the natural air $(\delta 180=40 \%$ o $)$ would also result in a $0.1 \mathrm{ppm}$ error in the reported value of the [626]. The difference between the 626 mole fractions that would have been derived without knowledge of the isotopic composition and those derived with this knowledge ranged from 0.06 to $0.12 \mathrm{ppm}$ and were therefore significant in this comparison. Errors in the reported 626 mixing ratios caused by other $\mathrm{CO}_{2}$ isotopologues were certainly less than $0.02 \mathrm{ppm}$. Hence, we ignored the isotopic composition variations of the less abundant isotopologues.

Table 2 shows the measurement results of ABC. We tested the ABC's potential accuracy by setting up three calibration cycles. In each cycle, two of the three tanks were alternatively measured for at least $5 \mathrm{~min}$ each. This alternation was 
repeated 10 times between Tank \#1 and \#2, 5 times between Tank \#1 and \#3, and 5 times between Tank \#2 and \#3. To analyze each cycle, one of the two tanks was selected to calibrate the quartz cell $\mathrm{CO}_{2}$ while the other one was treated as the sample tank. Three separate analyses of the same data were performed, with each analysis using a different tank as the basis for the calibration. ABC error estimations shown in Table 2 are the 1 standard deviation from repeated measurements. The differences between $\mathrm{ABC}$ measurements and the corrected Licor values for 626 mixing ratio are generally within $0.2 \mathrm{ppm}$.

\section{Summary and future development}

An $A B C$ prototype instrument measuring ambient dry air $\mathrm{CO}_{2}$ mixing ratio is developed in this study. The special features of this instrument are the use of the spectroscopic null method and the use of stable internal reference cells to replace the labor-intensive and uncertain calibration tanks. These features have been demonstrated to improve the instrument's real-time performance: one-second precision of $0.02 \mathrm{ppm} \mathrm{CO}_{2}$, long-term stability within $0.1 \mathrm{ppm}$ over one month and the potential accuracy of less than $0.2 \mathrm{ppm}$ without calibration while in use. The fast time-response, low drift and high precision of the $\mathrm{ABC}$ instrument are exceptional.

This work also implies the potential for spectroscopic measurements of other chemically inert atmospheric species without calibration tanks, but with equivalent or better measurement performances.

In future work, we plan to include water vapor mixing ratio using simultaneous $\mathrm{CO}_{2} / \mathrm{H}_{2} \mathrm{O}$ measurements for example near $3735.8 \mathrm{~cm}^{-1}$. The current instrument requires drying the sample which is a disadvantage for unattended remote monitoring. Spectral regions with co-existing $\mathrm{CO}_{2}$ and water absorption lines with appropriate line separations and line strengths are available. The additional spectral line broadening from the presence of water can be accounted for within the existing spectral analysis model. Successfully implementing this would eliminate the need to dry the sample gas stream.

Acknowledgements. BX thanks Elaine Gottlieb for calibrating the $\mathrm{CO}_{2}$ calibration tanks used in this study and Rick Wehr for providing the standard isotope tank. BX also thanks Gregory Santoni for advice on correcting isotope effects for Licor $\mathrm{CO}_{2}$ measurements. This work is funded by the DOE SBIR program under grant number DE-5C0000905.

Edited by: P. Werle

\section{References}

Andersen, P. C., Williford, C. J., David, D. E., and Birks, J. W.: Ultrasonic detector for high precision measurements of carbon dioxide, Anal. Chem., 82, 7929-7934, 2010.
Baer, D. S., Paul, J. B., Gupta, M., and O'Keefe, A.: Sensitive absorption measurements in the near-infrared region using off-axis integrated-cavity-output spectroscopy, Appl. Phys. B, 75, 261265, 2002.

Daube, B. C., Boering, K. A., Andrews, A. E., and Wofsy, S. C.: A high-precision fast-response airborne $\mathrm{CO}_{2}$ analyzer for in situ sampling from the surface to the middle stratosphere, J. Atmos. Ocean. Technol., 19, 1532-1543, 2002.

Engeln, R., Berden, G., Peeters, R., and Meijer, G.: Cavity enhanced absorption and cavity enhanced magnetic rotation spectroscopy, Rev. Sci. Inst., 69, 3763-3769, 1998.

Esler, M. B., Griffith, D. W. T., Wilson, S. R., and Steele, L. P.: Precision trace gas analysis by FT-IR spectroscopy. 1 . Simultaneous analysis of $\mathrm{CO}_{2}, \mathrm{CH}_{4}, \mathrm{~N}_{2} \mathrm{O}$, and $\mathrm{CO}$ in air, Anal. Chem., 72, 206-215, 2000.

Griffith, D. W. T., Deutscher, N. M., Caldow, C., Kettlewell, G., Riggenbach, M., and Hammer, S.: A Fourier transform infrared trace gas and isotope analyser for atmospheric applications, Atmos. Meas. Tech., 5, 2481-2498, doi:10.5194/amt-5-2481-2012, 2012.

Keeling, R. F., Manning, A. C., Paplawsky, W. J., and Cox, A. C.: On the long-term stability of reference gases for atmospheric $\mathrm{O}_{2} / \mathrm{N}_{2}$ and $\mathrm{CO}_{2}$ measurements, Tellus, 59B, 3-14, 2007.

Komhyr, W. D., Harris, T. B., Waterman, L. S., Chin, J. F. S., and Thoning, K. W.: Atmospheric carbon-dioxide at Mauna Loa Observatory. 1. NOAA Global monitoring for climatic-change measurements with a nondispersive infrared analyzer 1974-1985, J. Geophys. Res., 94, 8533-8547, 1989.

McManus, J. B., Nelson, D. D., Herndon, S. C., Shorter, J. H., Zahniser, M. S., Blaser, S., Hvozdara, L., Muller, A., Giovannini, M., and Faist, J.: Comparison of cw and pulsed operation with a TE-cooled quantum cascade infrared laser for detection of nitric oxide at $1900 \mathrm{~cm}^{-1}$, Appl. Phys. B, 85, 235-241, 2006.

McManus, J. B.: Paraxial matrix description of astigmatic and cylindrical mirror resonators with twisted axes for laser spectroscopy, Appl. Opt., 46, 472-482, 2007.

McManus, J. B., Zahniser, M. S., and Nelson, D. D.: Dual quantum cascade laser trace gas instrument with astigmatic Herriott cell at high pass number, Appl. Opt., 50, A74-85, 2011.

Nelson, D. D., McManus, J. B., Herndon, S. C., Shorter, J. H., and Zahniser, M. S.: Characterization of a near-room-temperature, continuous-wave quantum cascade laser for long-term, unattended monitoring of nitric oxide in the atmosphere, Opt. Lett., 31, 2012-2014, 2006.

Nelson, D. D., McManus, J. B., Herndon, S. C., Zahniser, M. S., Tuzson, B., and Emmenegger, L.: New method for isotopic ratio measurements of atmospheric carbon dioxide using a $4.3 \mathrm{um}$ pulsed quantum cascade laser, Appl. Phys. B, 90, 301-309, 2008.

Olivero, J. J. and Longbothum, R. L.: Empirical fits to Voigt line width: A brief review, J. Quant. Spectrosc. Ra., 17, 233-236, 1977.

Rella, C. W., Chen, H., Andrews, A. E., Filges, A., Gerbig, C., Hatakka, J., Karion, A., Miles, N. L., Richardson, S. J., Steinbacher, M., Sweeney, C., Wastine, B., and Zellweger, C.: High accuracy measurements of dry mole fractions of carbon dioxide and methane in humid air, Atmos. Meas. Tech., 6, 837-860, doi:10.5194/amt-6-837-2013, 2013. 
Stephens, B. B., Miles, N. L., Richardson, S. J., Watt, A. S., and Davis, K. J.: Atmospheric $\mathrm{CO}_{2}$ monitoring with singlecell NDIR-based analyzers, Atmos. Meas. Tech., 4, 2737-2748, doi:10.5194/amt-4-2737-2011, 2011.

Tittel, F. K., Richter, D., and Fried, A.: Mid-Infrared Laser Applications in Spectroscopy, in: Solid-State Mid-Infrared Laser Sources, edited by: Sorokina, I. T. and Vodopyanov, K. L., 89, 445-510, Springer-Verlag, 2003.

Tohjima, Y., Katsumata, K., Morino, I., Mukai, H., Machida, T., Akama, I., Amari, T., and Tsunogai, U.: Theoretical and experimental evaluation of the isotope effect of NDIR analyzer on atmospheric $\mathrm{CO}_{2}$ measurement, J. Geophys. Res., 114, D13302, doi:10.1029/2009JD011734, 2009.

Werle, P.: Time domain characterization of micrometeorological data based on a two sample variance, Agric. Forest Meteorol., 150, 832-840, 2010.

Werle, P.: Accuracy and precision of laser spectrometers for trace gas sensing in the presence of optical fringes and atmospheric turbulence, Appl. Phys. B, 102, 313-329, 2011.
Werle, P., Mücke, R., and Slemr, F.: The limits of signal averaging in atmospheric trace-gas monitoring by Tunable Diode-Laser Absorption Spectroscopy (TDLAS), Appl. Phys. B ,57, 131-139, 1993.

Wofsy, S. C., the HIPPO Science Team and Cooperating Modellers and Satellite Teams: HIAPER Pole-to-Pole Observations (HIPPO): fine-grained, global-scale measurements of climatically important atmospheric gases and aerosols, Phil. Trans. R. Soc. A, 369, 2073-2086, 2011.

Zhao, C. L. and Tans, P. P.: Estimating uncertainty of the WMO mole fraction scale for carbon dioxide in air, J. Geophys. Res., 111, D08S09, doi:10.1029/2005JD006003, 2006.

Zhou, L. X., Kitzis, D., and Tans, P.: Report of the 14th WMO Round-Robin Reference Gas Inter-comparison, 20022007, WMO/GAW Report No. 186, 2009. 